\section{Medical Principles and Practice}

Med Princ Pract 2019;28:236-241

DOI: $10.1159 / 000497612$
Received: July 8, 2018

Accepted: February 6, 2019

Published online: February 6, 2019

\title{
Pigment Epithelium-Derived Factor Affects Angiogenesis in Celiac Disease
}

\author{
Muhammet Yener Akpinar ${ }^{\mathrm{a}}$ Evrim Kahramanoglu Aksoy ${ }^{\mathrm{a}}$ \\ Ferdane Pirincci Sapmaz ${ }^{a}$ Ozlem Ceylan Dogan ${ }^{b}$ Metin Uzman ${ }^{a}$ \\ Yasar Nazligul ${ }^{a}$ \\ ${ }^{a}$ Department of Gastroenterology, Department of Medical Biochemistry, Kecioren Training and Research Hospital, \\ Ankara, Turkey; ${ }^{\mathrm{b}}$ Ankara University Medical Faculty, Ankara, Turkey
}

\section{Keywords}

Angiogenesis · Celiac disease · Pigment epithelium-derived factor

\section{Abstract}

Objective: Recent studies have demonstrated that angiogenesis is impaired in patients with celiac disease (CD). In this study, we evaluated the levels of the novel antiangiogenic factor pigment epithelium-derived factor (PEDF) in CD patients. Methods: Eighty-four patients were included in the study; 71 patients with CD and 13 healthy controls. In the CD patient cohort, there were 21 newly diagnosed patients, 19 with adherence to a gluten-free diet and 31 practicing no adherence to this diet. The PEDF levels were measured using enzyme-linked immunosorbent assays. Results: The data revealed that celiac patients had higher levels of PEDF than did healthy controls. PEDF levels were not significantly different among the three CD groups. Additionally, the PEDF levels were not correlated with tissue transglutaminase $\lg A$ or lgG. Conclusions: Our data indicate that PEDF levels are significantly higher in CD patients than those in the healthy controls. This result suggests that PEDF negatively affects angio- genesis in $\mathrm{CD}$. Although we did not observe any differences of PEDF levels among celiac patients, additional studies including more patients could clarify this issue.

(C) 2019 The Author(s)

Published by S. Karger AG, Basel

\section{Introduction}

Celiac disease (CD) is an immune-mediated enteropathy. Dietary gluten from wheat, rye, and barley triggers the immune system in genetically susceptible individuals and results in villous atrophy, intraepithelial lymphocytes, and crypt hyperplasia $[1,2]$. Not all individuals are symptomatic, and in adulthood, most patients are diagnosed incidentally, or they present with extraintestinal symptoms [3]. In recent years, since the clinicians raised their awareness of CD, diagnosis of CD has been increased. However, our knowledge regarding the physiopathology of CD has not increased. The majority of current studies have focused on the phenotype of immune reactions, oxidative stress, and immune system-related cytokines [4-6]. There is limited information regarding

\begin{tabular}{ll}
\hline KARGER & $\begin{array}{l}\text { (c) } 2019 \text { The Author(s) } \\
\text { Published by S. Karger AG, Basel }\end{array}$ \\
$\begin{array}{l}\text { E-Mail karger@karger.com } \\
\text { www.karger.com/mpp }\end{array}$ & $\begin{array}{l}\text { This is an Open Access article licensed under the Creative Commons } \\
\text { Attribution-NonCommercial-4.0 International License (CC BY-NC) } \\
\text { (http://www.karger.com/Services/OpenAccessicense), applicable to } \\
\text { the online version of the article only. Usage and distribution for com- } \\
\text { mercial purposes requires written permission. }\end{array}$
\end{tabular}

Muhammet Yener Akpinar

Kecioren Training and Research Hospital

Department of Gastroenterology, Sanatoryum Street

TR-06280 Kecioren, Ankara (Turkey)

E-Mail muhammet.yener@gmail.com 
the roles of angiogenesis and vascular pathology in CD. Cooke and Holmes [7] demonstrated that vascular architecture is disrupted in individuals with CD. More recently, Myrsky et al. [8] demonstrated that autoantibodies directed to transglutaminase disrupt angiogenesis. These results show that angiogenesis is disrupted and is diminished in CD. However, there is insufficient data to explain the role of angiogenesis in the physiopathology of CD.

Pigment epithelium-derived factor (PEDF) is a protein belonging to the serpin protease inhibitor family. It is commonly expressed in normal tissues and has multifunctional effects including antioxidant, antiangiogenic, anti-inflammatory, and neuroprotective properties $[9$, 10]. The antiangiogenic function is the leading characteristic feature of this protein, and PEDF has been shown to be the most potent endogenous negative regulator of angiogenesis. PEDF impacts angiogenesis in both healthy tissues and various tumor types [11] and it exerts its biologic effect by inhibiting vascular endothelial growth factor in different ways, resulting in decreased endothelial cell proliferation and tubule formation [12]. PEDF has been investigated in various conditions including myocardial infarction, diabetic nephropathy, and diabetic retinopathy, but there is no information on the role of PEDF in CD [13]. We hypothesized that PEDF may play a role in diminished angiogenesis in patients with CD.

\section{Subjects and Methods}

\section{Patient Selection and Study Design}

This is a prospective cross-sectional study conducted in Kecioren Training and Research Hospital during January-March 2018. All patients with CD including newly diagnosed and follow-up patients participated in the study based on their recent laboratory parameters. The patients were classified according to their time of diagnosis and consumption of a gluten-free diet (GFD).

(a) Newly diagnosed CD patients: These patients were newly diagnosed based on endoscopic, pathologic, and serologic tests.

(b) CD patients on GFD: These patients were selected from the $\mathrm{CD}$ patient pool and their serologic markers were negative. In this group, diagnosis was made at least 1 year previously.

(c) CD patients not on GFD: These patients were not compliant with GFD, and their serologic markers were positive. In this group, diagnosis was made at least 1 year previously.

(d) Control group: The control group consisted of healthy people without CD.

All endoscopic procedures were performed by experienced endoscopists. A duodenal biopsy was performed at least 4 times from different parts of the duodenum for diagnosis of $\mathrm{CD}$. Tissue transglutaminase IgA antibody (IgA anti-tTG) (>30 U/mL was positive) and tissue transglutaminase IgG antibody (IgG anti-tTG) $(>30 \mathrm{U} / \mathrm{mL}$ was positive) were used to support the diagnosis of CD. The patient's GFD compatibility was also used to support the diagnosis of CD.

Angiogenesis in Celiac Disease
Table 1. Demographic characteristics of patients

\begin{tabular}{ll}
\hline Clinical characteristics & Value \\
\hline Patients & 84 \\
$\quad$ Celiac disease & 71 \\
$\quad$ Controls & 13 \\
Age, years & $39.00 \pm 14.31$ \\
Male/female & $20(23.8 \%) / 64(76.2 \%)$ \\
Initial symptom & \\
$\quad$ Dyspepsia & $32(45.1 \%)$ \\
Diarrhea & $20(28.2 \%)$ \\
$\quad$ Anemia & $15(21.1 \%)$ \\
$\quad$ Malnutrition & $2(2.8 \%)$ \\
Screening & $1(1.4 \%)$ \\
$\quad$ Vitiligo investigation & $1(1.4 \%)$ \\
Median disease duration, months & $80.92 \pm 63.49$ \\
Family history & $2 / 71(2.8 \%)$ \\
\hline
\end{tabular}

\section{Assessment of PEDF}

Two milliliters of blood was collected from subjects who had read and signed the informed consent form to participate in the study. The samples were collected and centrifuged for $15 \mathrm{~min}$ at $2,500 \mathrm{~g}$ within a period of $30 \mathrm{~min}$ of collection. The blood samples were stored at $-80^{\circ} \mathrm{C}$ until the time of analysis. PEDF concentrations were measured using the sandwich enzyme-linked immunosorbent assay (ELISA) (Boster Technology, USA) Biological kit. The intra-assay coefficient for variation for high values was $4.2 \%$. The inter-assay coefficient for variation for high values was $7.4 \%$.

\section{Statistical Analysis}

The normality of distribution of continuous variables was tested by the Shapiro-Wilk test. The Mann-Whitney U test was used to compare 2 independent groups, and the Kruskal-Wallis test was used for all pairwise multiple comparison tests to compare more than 2 groups for non-normal data. The $\chi^{2}$ test was applied to investigate the relationship between 2 categorical variables, and the Spearman rank correlation coefficient was used for numerical variables. All statistical analyses were performed with SPSS for Windows version 24.0 , and a $p$ value $<0.05$ was accepted as statistically significant.

\section{Results}

Eighty-four subjects (71 patients with CD, 13 healthy controls) participated in our study. The mean age of our patients was $39.00 \pm 14.31$ years, and $76.2 \%$ of the patients were female. The most common initial symptom of patients was dyspepsia (45.1\%), followed by diarrhea (28.2\%) and anemia (21.1\%). Two of the seventy-one CD patients had a family history of $\mathrm{CD}$. The median disease duration was 324 months (12-324) except for the newly diagnosed CD patients (Table 1). 
Table 2. Comparison of demographic characteristics, initial symptoms, and laboratory parameters between the three groups

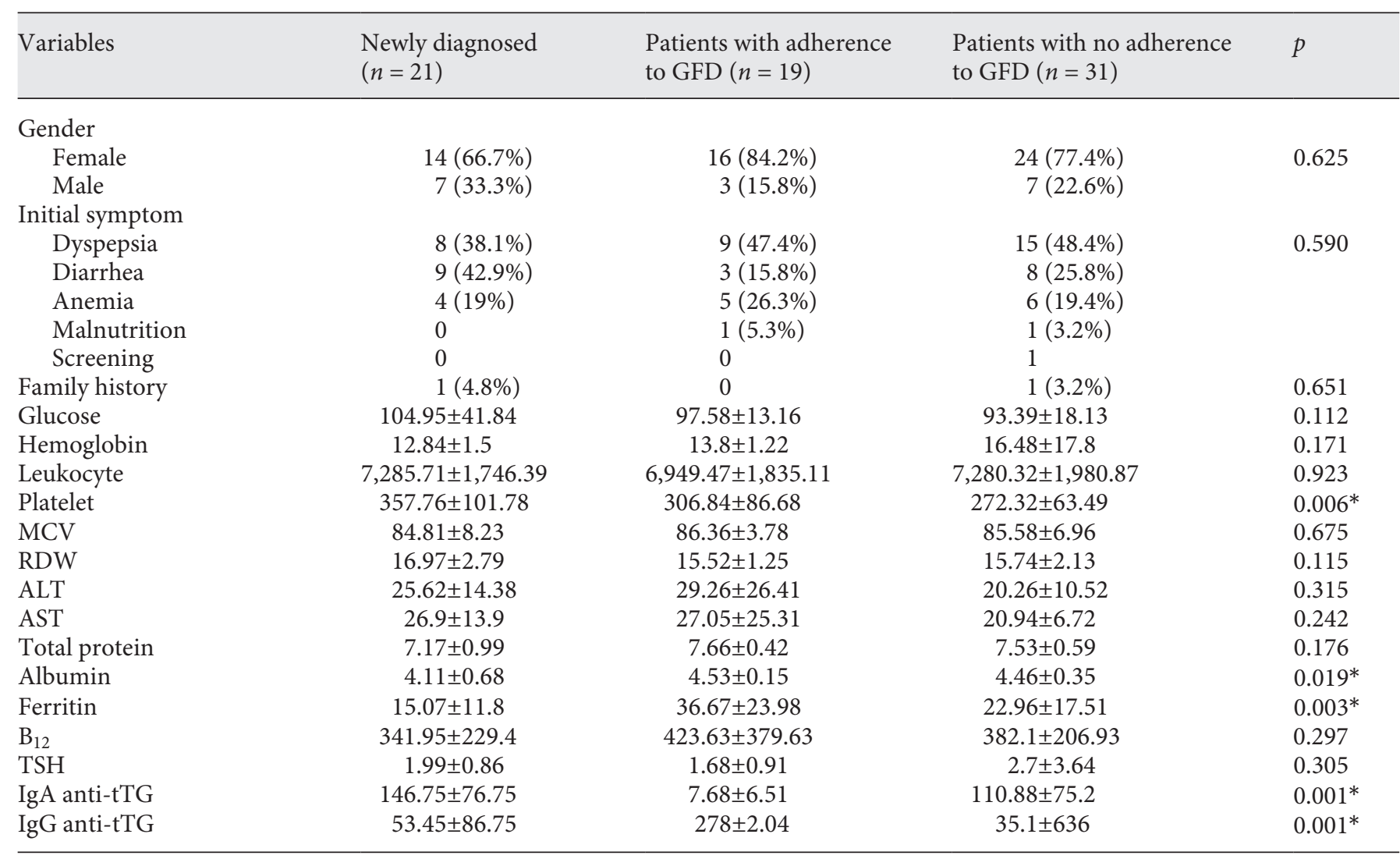

* Significantly different at the 0.05 level; the Kruskal-Wallis test was used.

Table 3. PEDF levels among the three groups

\begin{tabular}{|c|c|c|c|c|c|}
\hline PEDF level, pg/mL & $1,463.20(718.80-3,747.2)$ & $1,109.1(277.9-3,046.1)$ & $863.3(494.7-195,447)$ & $270.7(183.9-451.3)$ & $0.001^{*}$ \\
\hline
\end{tabular}

As described above, the $\mathrm{CD}$ patients were divided into the following three groups: newly diagnosed $(n=21)$, patients with adherence to GFD $(n=19)$, and patients with no adherence to GFD $(n=31)$. The control group and CD groups had an equal gender distribution. In the three CD groups, the gender, initial symptoms, and results of family screening were not significantly different. The laboratory parameters were investigated for the three groups, and no significantly different laboratory parameters were found except for albumin, ferritin, and platelet count. The ferritin levels were significantly lower in the newly diagnosed patients than in the other two groups. Additionally, the platelet count was significantly higher in the newly diagnosed CD patients than in patients not compatible with GFD (Table 2).

Levels of PEDF were significantly lower in the healthy control group than in CD patients regardless of whether they were newly diagnosed patients $(p=0.001)$ (Table 3; Fig. 1). Levels of PEDF were not significantly different among the three groups, which included $71 \mathrm{CD}$ patients. 
Fig. 1. PEDF levels were significantly higher in patients with CD compared to controls; nevertheless, PEDF levels were not different significantly between patients with CD.

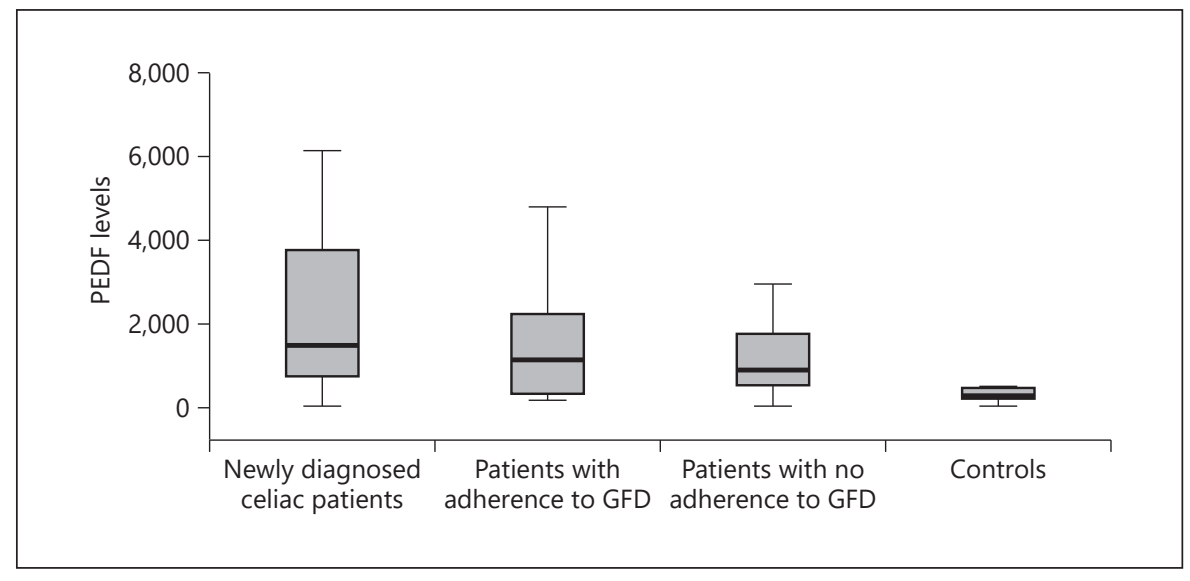

PEDF levels were not different between the newly diagnosed patients and those who were adherent to GFD ( $p=$ 0.178 ) or between newly diagnosed patients and those who were not adherent to GFD $(p=0.160)$. Furthermore, there was no difference in PEDF levels between the patients who were adherent to GFD and those who were not adherent to GFD $(p=0.919)$. There was no correlation between IgA anti-tTG and autoantibodies and serum levels of PEDF (Table 4).

\section{Discussion}

In this study, we demonstrated for the first time that PEDF levels are significantly higher in CD patients than in controls. We found that PEDF levels were not influenced by adherence to GFD. In addition, PEDF levels were not correlated with IgA anti-tTG or IgG anti-tTG antibodies that are antiangiogenic molecules.

The pathophysiology of CD is complex, and both the adaptive and innate immune system play important roles in addition to genetic and environmental factors. The inflammation observed in the small intestines in advanced stages of CD is a result of these factors. Inflammation also disrupts intestinal permeability, villous atrophy, and other sequelae of CD. Vascular biology including angiogenesis and endothelial dysfunction are not well described in $\mathrm{CD}$. There is limited information regarding the effects of this inflammation on angiogenesis in CD. Previous studies reported diminished angiogenesis in $\mathrm{CD}$, but it is unclear if the diminished angiogenesis is a result or a cause of $\mathrm{CD}$. Recent studies have described the angiogenic properties of several cytokines with pivotal roles in CD. One critical cytokine is IL-18, which promotes Th1 cells
Table 4. PEDF levels were not correlated with tissue transglutaminase IgA or IgG

\begin{tabular}{lll}
\hline Groups & IgA anti-tTG & IgG anti-tTG \\
\hline $\begin{array}{ll}\text { Newly diagnosed } \\
\text { PEDF level }\end{array}$ & \\
$\quad r$ & & \\
$p$ & 0.145 & 0.131 \\
$n$ & 0.530 & 0.573 \\
IgA anti-tTG & 21 & 21 \\
$r$ & & \\
$p$ & 1.000 & 0.521 \\
$n$ & & 0.015 \\
\end{tabular}

Patients with adherence to GFD

PEDF level

\begin{tabular}{cll}
$r$ & 0.117 & 0.227 \\
$p$ & 0.634 & 0.349 \\
$n$ & 19 & 19 \\
IgA anti-tTG & & \\
$r$ & 1.000 & 0.388 \\
$p$ & & 0.101 \\
$n$ & 19 & 19 \\
\hline
\end{tabular}

Patients with no adherence to GFD

PEDF level

$\begin{array}{ccl}r & -0.018 & 0.281 \\ p & 0.922 & 0.126 \\ n & 31 & 31 \\ \text { IgA anti-tTG } & & \\ r & 1.000 & 0.227 \\ p & & 0.220 \\ n & 31 & 31\end{array}$

$r$, Spearman correlation factor. 
and influences the inflammatory response within the small intestine [14]. Park et al. [15] demonstrated that IL18 is an angiogenic mediator both in vivo and in vitro. IL-15 is another important cytokine that promotes lymphocyte signaling and survival, leading to villous atrophy [16]; it also promotes angiogenesis in different conditions [17]. However, the angiogenic properties of these cytokines have not been demonstrated in patients with CD. Here, we demonstrated that both newly diagnosed patients and those following GFD had higher levels of PEDF than the healthy controls. This result is consistent with the published literature showing that angiogenesis is diminished in CD. We suggest that PEDF and other as yet unidentified molecules could affect angiogenesis in CD. Diminished angiogenesis is an important process in CD. Even though it is not the trigger event in physiopathology, it can be a result, and also a cause, of both prolonged inflammation and tissue damage in CD.

Autoantibodies against tissue transglutaminase 2 (TG2) are a hallmark of the diagnosis and pathophysiology of CD. TG2 is an enzyme within the transglutaminase family and normally localizes to the intracellular space. In $\mathrm{CD}, \mathrm{TG} 2$ converts glutamine to glutamate, which is an antigenic precursor that stimulates immune reactions. The role of anti-TG2 autoantibodies in CD was unclear until recently. The study by Matysiak-Budnik et al. [18] showed that autoantibodies increase the permeability of the intestine to glutamine. Additionally, large quantities of anti-tTG2 predict the severity of pathologic stages. We know that anti-TG2 antibodies inhibit angiogenesis [8, 19]. Although antiapoptotic properties of anti-tTG2 antibodies were demonstrated, we do not understand the relationship between anti-tTG2 antibodies and proangiogenic and antiangiogenic molecules. In this study, we evaluated the relationship between tTG2 and PEDF and found there was no correlation between PEDF and autoantibodies.

Our study has several limitations. Although determining the levels of PEDF can suggest a potential role for $\mathrm{PEDF}$ in $\mathrm{CD}$, immunohistochemical staining or PCR- based studies will provide more information on the role of PEDF in CD. Although we concluded that determination of tTG is enough to identify our study groups, some patients with the normal range of tTG may have persistent inflammatory changes in the affected segment/s of the intestine. Thus, some CD patients on GFD group and normal tTG may not have a histological response. This knowledge can explain the slight elevation of PEDF in CD patients on GFD compared to CD patients who are not on GFD. The available literature on PEDF indicates that certain conditions can affect the serum levels of PEDF. Renal insufficiency is one regulator of serum levels of PEDF, but others are not known. Another limitation of this study is the limited number of patients. Additional studies with larger patient cohorts are needed to determine the molecules that potentially influence angiogenesis. Nevertheless, it is clear that angiogenesis is impaired in $\mathrm{CD}$; future studies will elucidate the steps in this process.

\section{Conclusion}

Our study shows that individuals newly diagnosed with CD as well as those on GFD have higher levels of PEDF as compared to healthy controls. PEDF may contribute to the pathophysiology of CD by diminishing angiogenesis.

\section{Statement of Ethics}

The study protocol was approved by the Ethics Committee of Kecioren Training and Research Hospital. All individuals gave written informed consent.

\section{Disclosure Statement}

The authors declare that they have no conflict of interest to disclose.

\section{References}

1 Singh P, Arora A, Strand TA, Leffler DA, Catassi C, Green PH, et al. Global Prevalence of Celiac Disease: Systematic Review and Metaanalysis. Clin Gastroenterol Hepatol. 2018 Jun;16(6):823-836.e2.

2 Emilsson L, Lebwohl B, Green PH, Murray JA, Mårild K, Ludvigsson JF. Mucosal healing and the risk of serious infections in patients with celiac disease. United European Gastroenterol J. 2018 Feb;6(1):55-62.
3 Mahadev S, Laszkowska M, Sundström J, et al. Prevalence of Celiac Disease in Patients with Iron Deficiency Anemia - A Systematic Review with Meta-analysis. Gastroenterology. 2018 Aug; 155(2):374-382.e1.

4 Saccomanno D, Tomba C, Magri F, Backelandt P, Roncoroni L, Doneda L, et al. Antisulfatide reactivity in patients with celiac disease. Scand J Gastroenterol. 2017 Apr;52(4): 409-13. 
5 Attarwala $\mathrm{H}$, Clausen V, Chaturvedi P, Amiji MM. Cosilencing Intestinal Transglutaminase- 2 and Interleukin- 15 Using Gelatin-Based Nanoparticles in an in Vitro Model of Celiac Disease. Mol Pharm. 2017 Sep;14(9):303644.

6 Rashidiani S, Jalili A, Babaei E, Sheikhesmaeili F, Fakhari S, Ataee P, et al. The chemokine CCL28 is elevated in the serum of patients with celiac disease and decreased after treatment. Am J Clin Exp Immunol. 2017 Jun; 6(4):60-5.

7 Cooke WT, Holmes GK. Coeliac disease. Edinburgh: Churchill Livingstone; 1984.

8 Myrsky E, Kaukinen K, Syrjänen M, Korponay-Szabó IR, Mäki M, Lindfors K. Coeliac disease-specific autoantibodies targeted against transglutaminase 2 disturb angiogenesis. Clin Exp Immunol. 2008 Apr;152(1): 111-9.

9 Bilak MM, Corse AM, Bilak SR, Lehar M, Tombran-Tink J, Kuncl RW. Pigment epithelium-derived factor (PEDF) protects motor neurons from chronic glutamate-mediated neurodegeneration. J Neuropathol Exp Neurol. 1999 Jul;58(7):719-28.
10 Sawant S, Aparicio S, Tink AR, Lara N, Barnstable CJ, Tombran-Tink J. Regulation of factors controlling angiogenesis in liver development: a role for PEDF in the formation and maintenance of normal vasculature. Biochem Biophys Res Commun. 2004 Dec;325(2):40813.

11 Ek ET, Dass CR, Contreras KG, Choong PF. Inhibition of orthotopic osteosarcoma growth and metastasis by multitargeted antitumor activities of pigment epithelium-derived factor. Clin Exp Metastasis. 2007;24(2):93-106.

12 Cai J, Jiang WG, Grant MB, Boulton M. Pigment epithelium-derived factor inhibits angiogenesis via regulated intracellular proteolysis of vascular endothelial growth factor receptor 1. J Biol Chem. 2006 Feb;281(6): 3604-13.

13 Liang H, Hou H, Yi W, Yang G, Gu C, Lau $\mathrm{WB}$, et al. Increased expression of pigment epithelium-derived factor in aged mesenchymal stem cells impairs their therapeutic efficacy for attenuating myocardial infarction injury. Eur Heart J. 2013 Jun;34(22):1681-90.

14 Zupin L, Catamo E, Polesello V, Crovella S, Segat L. Interleukin-18 gene promoter polymorphisms and celiac disease in Italian patients. Mol Biol Rep. 2015 Feb;42(2):525-33.
15 Park CC, Morel JC, Amin MA, Connors MA, Harlow LA, Koch AE. Evidence of IL-18 as a novel angiogenic mediator. J Immunol. 2001 Aug;167(3):1644-53.

16 Escudero-Hernández C, Plaza-Izurieta L, Garrote JA, Bilbao JR, Arranz E; CEGEC. Association of the IL-15 and IL-15Ra genes with celiac disease. Cytokine. 2017 Nov;99:73-9.

17 Rohena-Rivera K, Sánchez-Vázquez MM, Aponte-Colón DA, Forestier-Román IS, Quintero-Aguiló ME, Martínez-Ferrer M. IL15 regulates migration, invasion, angiogenesis and genes associated with lipid metabolism and inflammation in prostate cancer. PLoS One. 2017 Apr;12(4):e0172786.

18 Matysiak-Budnik T, Moura IC, Arcos-Fajardo M, Lebreton C, Ménard S, Candalh C, et al. Secretory IgA mediates retrotranscytosis of intact gliadin peptides via the transferrin receptor in celiac disease. J Exp Med. 2008 Jan; 205(1):143-54.

19 Caja S, Myrsky E, Korponay-Szabo IR, Nadalutti C, Sulic AM, Lavric M, et al. Inhibition of transglutaminase 2 enzymatic activity ameliorates the anti-angiogenic effects of coeliac disease autoantibodies. Scand J Gastroenterol. 2010 Apr;45(4):421-7. 